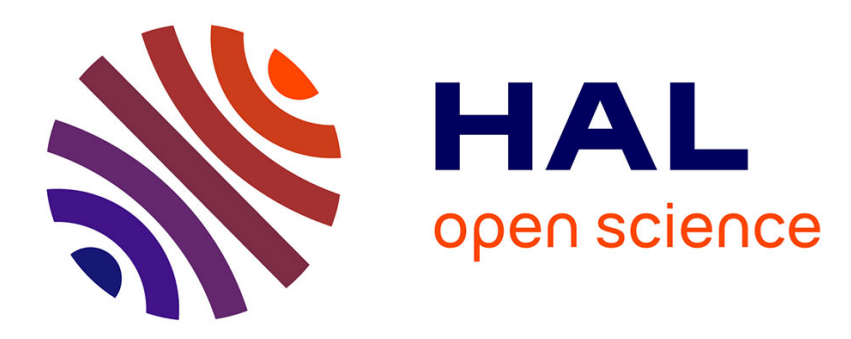

\title{
Numerical Modeling of the Ductile-Brittle Transition
}

A. Needleman

\section{To cite this version:}

A. Needleman. Numerical Modeling of the Ductile-Brittle Transition. Journal de Physique IV Proceedings, 1996, 06 (C6), pp.C6-325-C6-334. 10.1051/jp4:1996632 . jpa-00254460

\section{HAL Id: jpa-00254460 https://hal.science/jpa-00254460}

Submitted on 1 Jan 1996

HAL is a multi-disciplinary open access archive for the deposit and dissemination of scientific research documents, whether they are published or not. The documents may come from teaching and research institutions in France or abroad, or from public or private research centers.
L'archive ouverte pluridisciplinaire HAL, est destinée au dépôt et à la diffusion de documents scientifiques de niveau recherche, publiés ou non, émanant des établissements d'enseignement et de recherche français ou étrangers, des laboratoires publics ou privés. 


\title{
Numerical Modeling of the Ductile-Brittle Transition
}

\author{
A. Needleman \\ Division of Engineering, Brown University, Providence, RI, 02912, U.S.A.
}

\begin{abstract}
Numerical studies of the ductile-brittle transition are described that are based on incorporating physically based models of the competing fracture mechanisms into the material's constitutive relation. An elastic-viscoplastic constitutive relation for a porous plastic solid is used to model ductile fracture by the nucleation and subsequent growth of voids to coalescence. Cleavage is modeled in terms of attaining a temperature and strain rate independent critical value of the maximum principal stress over a specified material region. Various analyses of ductile-brittle transitions carried out within this framework are discussed. The fracture mode transition emerges as a natural outcome of the initial/boundary value problem solution.
\end{abstract}

\section{INTRODUCTION}

The competition between the micro-mechanisms of ductile void growth and brittle cleavage plays an important role in determining the macroscopic fracture resistance of structural steels. High fracture resistance is associated with the ductile mechanism and low fracture resistance with cleavage. The onset of brittle fracture is often the critical event that limits the load bearing capacity of the structure or component. Hence, the considerable interest in being able to predict this fracture mode transition.

A framework for modeling the ductile-brittle transition associated with a change in fracture mechanism from plastic void growth to cleavage cracking has been developed in Tvergaard and Needleman [1]. The basis for the void growth modeling is a plastic flow rule for progressively cavitating solids due to Gurson [2] and the cleavage model draws on the critical stress over a critical distance criterion of Ritchie, Knott and Rice [3].

Various analyses of ductile-brittle transitions carried out within this framework are discussed. Analyses of the Charpy V-notch test and tensile cracks in small scale yielding are considered where a normal ductile-brittle transition occurs; ductile failure at low loading rates (or high temperatures) and brittle failure at high loading rates (or low temperatures). Under dynamic mode II loading, a reverse transition is observed, Kalthoff [4], in that brittle failure occurs at low loading rates and ductile failure at high loading rates. A mechanism for this seemingly paradoxical behavior emerges from calculations carried out within this framework, Needleman and Tvergaard [15]. Convergence of numerical solutions is illustrated when relevant characteristic lengths are incorporated into the formulation. 


\section{FIELD EQUATIONS}

The analyses discussed employ a Lagrangian formulation of the field equations, with full account taken of finite strains and rotations. The position of each material particle is labelled in a conveniently chosen reference configuration and these labels, together with time, serve as the set of independent variables. The labels can be identified with the position of the given particle relative to the origin of a fired Cartesian frame and this position is denoted by $\mathbf{x}$.

In the current configuration the material point initially at $\mathbf{x}$ is at $\overline{\mathbf{x}}$. The displacement vector $\mathbf{u}$ and the deformation gradient $\mathbf{F}$ are defined by

$$
\mathbf{u}=\overline{\mathbf{x}}-\mathbf{x} \quad \mathbf{F}=\frac{\partial \overline{\mathbf{x}}}{\partial \mathbf{x}}
$$

The rate of deformation tensor is defined by

$$
\mathbf{d}=\frac{1}{2}\left(\dot{\mathbf{F}} \cdot \mathbf{F}^{-1}+\mathbf{F}^{-T} \cdot \dot{\mathbf{F}}^{T}\right)
$$

where ()$^{-T}$ is the inverse transpose, $\mathbf{a} \cdot \mathbf{b}=a^{i} b_{i}$ and $\left({ }^{\circ}\right)$ is $\partial() / \partial t$.

The momentum balance is expressed in terms of the nonsymmetric nominal stress tensor $\mathbf{s}$, which is related to the force, $d \mathbf{f}$, transmitted across a material element of area $d S$ and orientation $\mathbf{n}$ in the reference configuration by $d \mathbf{f}=\mathbf{n} \cdot \mathbf{s} d S$

With tractions prescribed over part of the surface and displacements on the remainder, the dynamic principle of virtual work is written as

$$
\int_{V} \mathbf{s}: \delta \mathbf{F} d V=\int_{S} \mathbf{T} \cdot \delta \mathbf{u} d S-\int_{V} \rho \frac{\partial^{2} \mathbf{u}}{\partial t^{2}} \cdot \delta \mathbf{u} d V
$$

where $V, S$ and $\rho$ are the volume, surface and mass density, respectively, of the body in the reference configuration, and $\mathbf{A}: \mathbf{B}=A^{i j} B_{j i}$.

Inelastic constitutive relations are expressed in terms of the symmetric Cauchy, $\boldsymbol{\sigma}$, or Kirchhoff, $\tau$, stress measures which are related to $\mathrm{s}$ through

$$
\mathbf{s}=\mathbf{F}^{-1} \cdot \boldsymbol{\tau}=\operatorname{det}(\mathbf{F}) \mathbf{F}^{-1} \boldsymbol{\sigma}
$$

where $\operatorname{det}(\mathbf{F})$ is the ratio of the volume of a material element in the current configuration to its volume in the reference configuration.

\section{MATERIAL MODEL}

The basis for the material model is a viscoplastic version, Pan et al. [5], of Gurson's [2] constitutive relation for porous plastic solids. The rate of deformation is written as the sum of an elastic part (small elastic strains are assumed), a plastic part and a part due to thermal straining,

$$
\mathbf{d}=\mathbf{d}^{e}+\mathbf{d}^{p}+\mathbf{d}^{T}
$$

with

$$
\mathbf{d}^{e}=\frac{1+\nu}{E} \hat{\boldsymbol{\sigma}}-\frac{\nu}{E}(\hat{\boldsymbol{\sigma}}: \mathbf{I}) \mathbf{I} \quad \mathbf{d}^{T}=\alpha \dot{T} \mathbf{I}
$$

where $\hat{\sigma}$ is the Jaumann rate of Cauchy stress, $T$ is the temperature, $\alpha$ is the thermal expansion coefficient, $E$ is Young's modulus, $\nu$ is Poisson's ratio and $I$ is the identity tensor.

The plastic flow rule is based on the potential

$$
\Phi=\frac{\sigma_{e}^{2}}{\bar{\sigma}^{2}}+2 q_{1} f^{*} \cosh \left(\frac{3 q_{2} \sigma_{h}}{2 \bar{\sigma}}\right)-1-q_{1}\left(f^{*}\right)^{2}=0
$$


Here, $\tilde{\sigma}$ is the matrix flow strength and

$$
\boldsymbol{\sigma}^{\prime}=\boldsymbol{\sigma}-\sigma_{h} \mathbf{I} \quad \sigma_{e}^{2}=\frac{3}{2} \boldsymbol{\sigma}^{\prime}: \boldsymbol{\sigma}^{\prime} \quad \sigma_{h}=\frac{1}{3}(\boldsymbol{\sigma}: \mathbf{I})
$$

With $f^{*} \equiv 0,(7)$ reduces to the Mises potential. The parameters $q_{1}$ and $q_{2}$ were introduced in $[7,8]$ to bring predictions of the model into closer agreement with full numerical analyses of a periodic array of voids. The bilinear function $f^{*}(f)$ was introduced in [9] to account for the effects of rapid void coalescence at failure

$$
f^{*}=\left\{\begin{array}{c}
f f<f_{c} \\
f_{c}+\left(f_{u}^{*}-f_{c}\right) /\left(f_{f}-f_{c}\right) \quad f \geq f_{c}
\end{array}\right.
$$

where $f$ is the void volume fraction. The constant $f_{u}^{*}=1 / q_{1}$ is the value of $f^{*}$ at zero stress. As $f \rightarrow f_{f}, f^{*} \rightarrow f_{u}^{*}$ and the material loses all stress carrying capacity.

The plastic part of the rate of deformation, $\mathbf{d}^{p}$, is obtained as

$$
\mathbf{d}^{p}=\left[\frac{(1-f) \overline{\boldsymbol{\sigma}} \dot{\bar{\epsilon}}}{\boldsymbol{\sigma}: \frac{\partial \phi}{\partial \boldsymbol{\sigma}}}\right] \frac{\partial \phi}{\partial \boldsymbol{\sigma}}
$$

For a power law strain rate hardening matrix

$$
\dot{\bar{\epsilon}}=\dot{\bar{\epsilon}}_{0}\left(\frac{\bar{\sigma}}{g(\bar{\epsilon}, T)}\right)^{1 / m}
$$

The function $g(\bar{\epsilon}, T)$, with $\bar{\epsilon}=\int \dot{\bar{\epsilon}} d t$, represents the (temperature dependent) effective stress versus effective strain response in a tensile test carried out at a strain rate $\dot{\bar{\epsilon}}=\dot{\bar{\epsilon}}_{0}$ and $m$ is the strain rate hardening exponent.

The rate of increase of void volume fraction in general has contributions from both the growth of existing voids and from the nucleation of new voids

$$
\dot{f}=\dot{f}_{\text {growth }}+\dot{f}_{\text {nucleation }}
$$

The contribution from the growth of existing voids is determined from the con 1 tion that plastic deformation of the matrix is volume preserving so that

$$
\dot{f}=(1-f) \mathrm{d}^{p}: \mathbf{I}
$$

The contribution from the nucleation of new voids is taken to be either strain controlled or stress controlled. In either case, void nucleation is taken to follow a normal distribution as suggested by Chu and Needleman [6].

Due to the presence of micro-voids, the plastic flow is dilational, and pressure sensitive. Although the matrix material continues to harden, the aggregate can soften, with the stress carrying capacity of the aggregate eventually vanishing.

Brittle cleavage is described in terms of a temperature and strain rate independent critical value of the maximum principal stress being reached over a specified material region called a cleavage grain, i.e.,

$$
\frac{1}{V_{g r}} \int_{V_{g r}} \sigma_{I} d V=\sigma_{c}
$$

where $V_{g r}$ is the cleavage grain volume, $\sigma_{I}$ is the maximum principal stress and $\sigma_{c}$ is the temperature and strain rate independent critical cleavage stress. Different values of the critical cleavage stress may be specified for each cleavage grain.

The basis for the change in fracture mechanism is that ductile fracture by plastic void growth requires large strains, while brittle fracture occurs when the stress level is high enough to cause fracture in a cleavage grain. Since the flow strength decreases with increasing temperature and decreasing strain rate, at high temperatures and low strain rates, plastic flow limits the achievable stress levels so that cleavage does not occur before the large strains needed for ductile fracture are achieved. At lower temperatures or higher strain rates, the stress level required for cleavage can be reached before there is extensive plastic flow and fracture takes place in a brittle manner. 

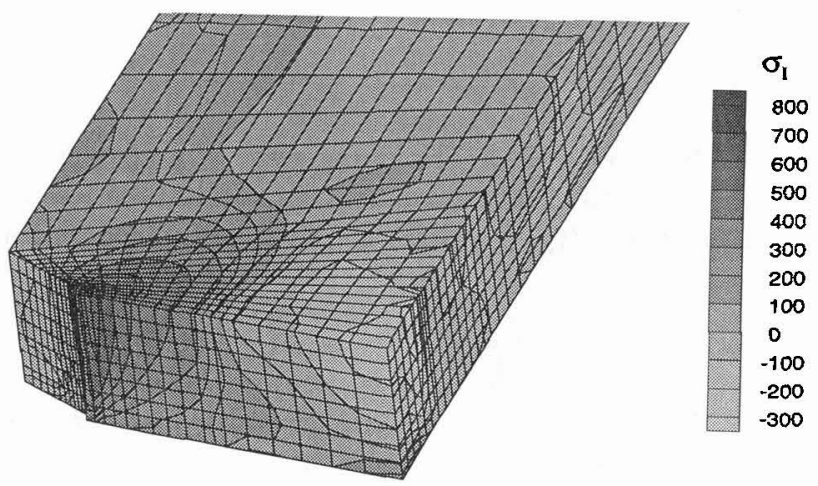

(a)

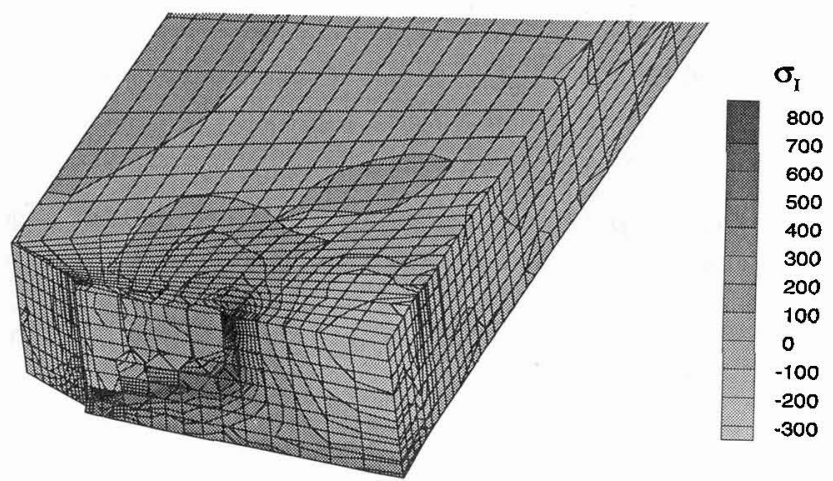

(b)

Figure 1: Contours of maximum principal stress for (a) ductile failure and (b) brittle failure.

\section{NUMERICAL RESULTS}

In two dimensions the finite element meshes are built up of four 'crossed' linear displacement elements, while in three dimensions twenty node bricks with $2 \times 2 \times 2$ reduced integration are used. In the dynamic analyses discussed, explicit time integration is employed. The rate dependent constitutive relations are integrated using the rate tangent modulus method of Peirce et al. [10] and material failure is implemented by the element vanish technique of Tvergaard [11]. Further information on the numerical methods used is given in the references cited.

\subsection{Charpy impact test}

Although the Charpy specimen is square and is not necessarily very wide compared to the dimensions of the notch region undergoing large plastic straining, analyses of the Charpy impact test have been carried out assuming plane strain conditions. Comparison of a full three dimensional analysis and a two dimensional plane strain approximation for the Charpy V-notch test using an isotropically hardening solid with no damage, Mathur et al. [12], has shown that the average force level and the absorbed energy predicted by the two dimensional analysis is somewhat too high. These analyses 
also showed that there are rather strong edge effects, but that there is a region near the central plane of the specimen where relevant field quantities are nearly constant.

In Mathur et al. [13] ductile failure by the nucleation and growth of micro voids to coalescence and for crack growth by a cleavage mechanism were both accounted for, allow for a direct evaluation of the usefulness of failure mode predictions obtained by planar analyses. The presumption in the calculations in Mathur et al. [13] is that the high stresses and strains induced by the Charpy Vnotch occur over a region that is large compared to relevant microstructural length scales such as the cleavage grain size or the spacing of void nucleating inclusions. Hence, such microstructural length scales are not explicitly accounted for in the modeling.

The initial trends in Mathur et al. [13] agreed rather well with those in Mathur et al. [12], but as the crack extends from the notch tip, the differences between the three dimensional predictions and the two dimensional plane strain approximation increased. A significant amount of crack tunneling developed in the range of material behavior where cleavage plays an important role. For ductile failure the difference is more like that expected based on the results with no damage, but there is still more crack growth in the central part of the specimen than near the free edge.

Figure 1, from results in Mathur et al. [13], shows contours of maximum principal stress (in MPa) exhibiting the mode of crack growth in a Charpy impact test for (a) ductile failure at $200 \mu \mathrm{s}$ and (b) brittle failure at $100 \mu \mathrm{s}$. Crack growth is occurring along the $z=0$ plane, which is the notch root plane. The plane $y=0$, at the top in Fig. 1, is the mid-thickness plane. Symmetry about $z=0$ and $y=0$ is assumed in the calculations. Corresponding plane strain analyses predicted more rapid crack growth at the central part of the notch than found by the three dimensional analyses. A planar analysis cannot represent important three dimensional effects, such as the ductile failure region at the free surface edge of a crack growing mainly by the brittle mechanism, or the reduced constraint due to pull-in of the free surface in the notch region as illustrated in Fig. 1.

\subsection{Mode I crack}

At a crack tip, substantial gradients of stress and strain occur over distances that are of the order of, or smaller than, microstructurally relevant length scales. Hence, such length scales need to be accounted for in the modeling. Tvergaard and Needleman [1] and Gao et al. [14] have carried out analyses of the competition between cleavage fracture and ductile tearing in structural steels in the ductile-brittle transition region. In both studies, two dimensional plane strain analyses were carried out. A small initial crack opening was accounted for and two populations of void nucleating particles were represented, i.e. large weak inclusions that nucleate voids at an early stage, and small strong particles that require large strains for void nucleation. The large inclusions were modeled as "islands" of stress controlled nucleation while the small particles (carbides in steels) were taken to be uniformly distributed.

In order to predict fracture toughness, a material length scale needs to enter the initial/boundary value problem formulation, if only from dimensional considerations. For the ductile fracture mechanism, the key material length scale is the spacing between the large inclusions. For the brittle cleavage mechanism, the cleavage grain size provides the length scale.

A main focus in Gao et al. [14] was on the amount of ductile crack growth that occurs before the transition to cleavage as a function of temperature and constraint. Gao et al. [14] presented results for the effect of mesh resolution on the crack growth predictions for both the ductile and brittle mechanisms. The effect of element size (near the crack tip) on the computed crack growth response is shown in Figs. 2 and 3, taken from [14]. Figure 2 shows plots of crack advance $\Delta a$ versus time at four temperature. At the two lower temperatures, where the failure mode is mainly cleavage, the crack growth rates are essentially the same for the two discretizations. When the temperature is increased, and ductile fracture predominates, the sensitivity to the discretization increases. Crack initiation is delayed for the coarser mesh, but the initial crack speed is greater with the coarser mesh. For the purely ductile case in Fig. 3 results for three meshes are shown. Here, a normalized value of 


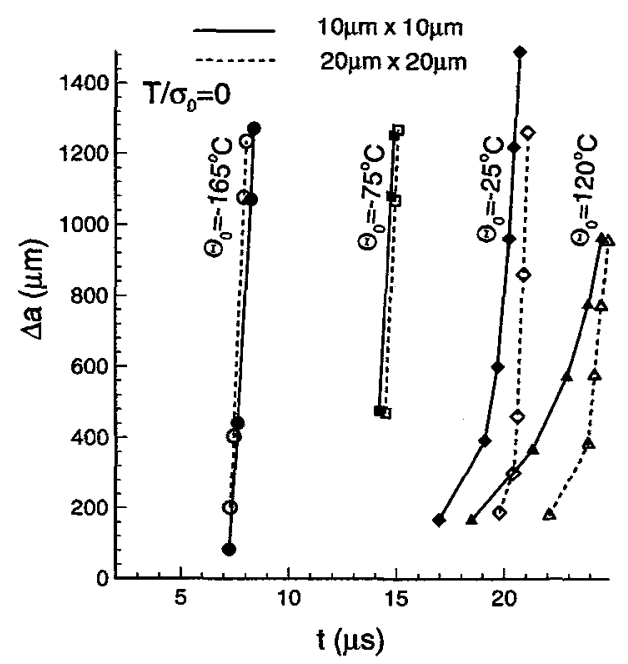

Figure 2: Crack length versus time for two meshes.

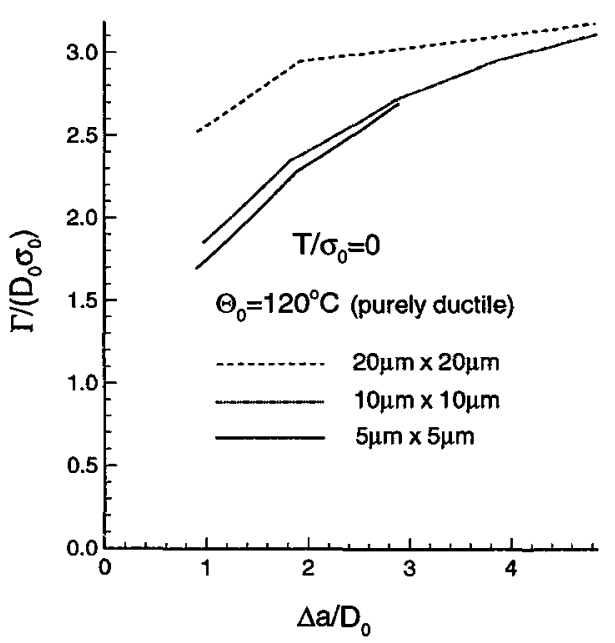

Figure 3: Crack growth resistance curves for three meshes. 


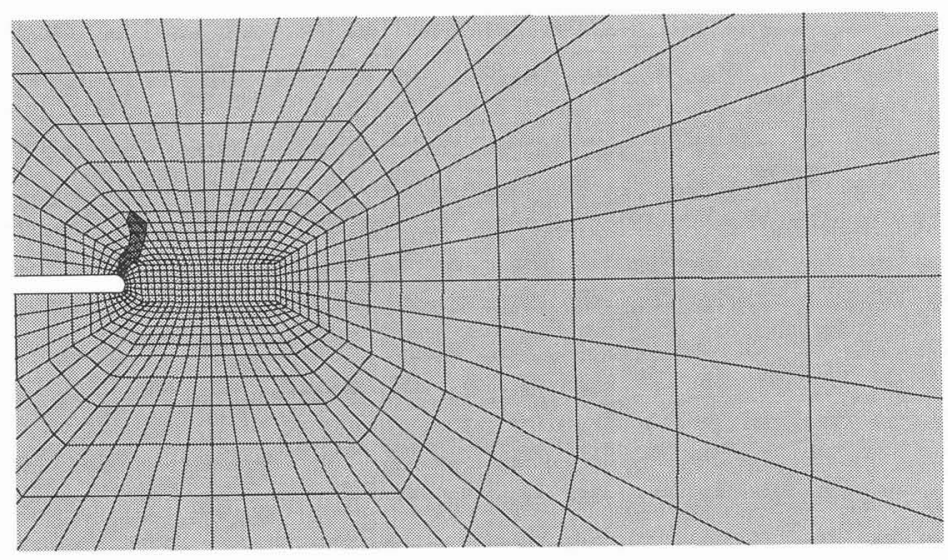

(a)

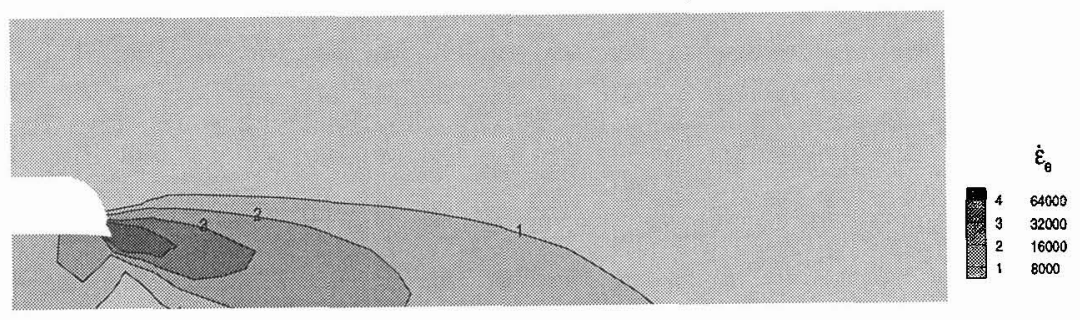

Figure 4: Contour plots showing the predicted directions of crack propagation. (a) shows the low loading rate failure mode and has the finite elements where cleavage occurred darkened. (b) shows contours of constant strain rate indicating the direction of localization associated with the high loading rate failure mode.

the material's crack growth resistance $\Gamma$ (the total work of fracture per unit area of crack advance) is plotted against the normalized amount of crack growth $\Delta a$. Figures 2 and 3 illustrate that by explicitly incorporating relevant microstructural lengths into the boundary value problem formulation convergent solutions for both the ductile and brittle modes of crack growth can be obtained.

\subsection{Mode II crack}

An experimental technique for subjecting edge cracks in plate specimens to high rates of shear loading was proposed by Kalthoff [4]. The method relies on impacting a plate by a cylindrical projectile between two parallel edge cracks. In experiments on a high-strength steel it was observed that at low impact velocities a brittle cleavage fracture occurred, whereas at sufficiently high impact velocities the failure involved shear localization leading to ductile fracture (of course, for low enough impact velocities there is no fracture). This observed fracture mode transition is, of course, exactly the opposite of the usual brittle-ductile transition behavior and seemed paradoxical.

Needleman and Tvergaard [15] carried out an analysis of the Kalthoff experiment using the 
framework described above. The calculations indicate that the observed behavior is mainly the outcome of a competition between thermal softening induced localization and cleavage. Porosity induced softening appears to play an important, but secondary, role. Because adiabatic conditions are assumed, the porosity is uniformly distributed and the critical stress for cleavage is spatially uniform, no material length scale enters the formulation. Accordingly, the results in [15] are confined to the initiation of fracture. A geometrical length, the crack tip radius, is present in the model and does affect the stress and strain distributions near the crack tip. Kalthoff [4] reported that the projectile velocity at which the brittle-ductile transition occurred depended on the initial crack tip radius.

Figure 4, taken from [15], illustrates the low rate, Fig. 4a, and high rate, Fig. 4b, failure modes. There are two regions with the greatest straining; directly ahead of the initial crack and at about $-135^{\circ}$ from the initial crack plane. The negative hydrostatic stress below the initial crack plane restricts void growth to the high strain region ahead of the initial crack. The greatest tensile stress is above the initial crack plane. The straining ahead of the initial crack increases more rapidly with impact velocity than does the tensile stress magnitude above the initial crack. Flow localization due to thermal and porosity induced softening can occur, which then reduces the tensile stress magnitude above the initial crack. This is what gives brittle fracture at low impact velocities, and ductile fracture at high impact velocities as seen in Fig. 4. The seeming paradoxical type of ductile-brittle transition observed by Kalthoff [4] is directly predicted by the calculations in [15]. Thus, the same theoretical framework predicts both the expected ductile-brittle transition mode under tensile loading conditions and the anomalous ductile-brittle transition observed by Kalthoff [4].

\section{Acknowledgement}

The support of the Office of Naval Research through grant N00014-89-J-3054 is gratefully acknowledged.

\section{References}

[1] Tvergaard, V., Needleman, A., Int. J. Fract. 59 (1993) 53-67.

[2] Gurson, A.L., Plastic Flow and Fracture Behavior of Ductile Materials Incorporating Void Nucleation, Growth and Interaction, Ph.D Thesis 1975, Brown University.

[3] Ritchie, R.O., Knott, J.F., Rice, J.R., J. Mech. Phys. Solids 21 (1973) 395-410.

[4] Kalthoff, J.K., Appl. Mech. Rev. 43 (1988) S247-S250.

[5] Pan, J., Saje, M., Needleman, A., Int. J. Fract. 21 (1983) 261-278.

[6] Chu, C.C., Needleman, A., J. Engin. Mat. Tech. 102 (1980) 249-256.

[7] Tvergaard, V., Int. J. Fract. 17 (1981) 389-407.

[8] Tvergaard, V., Int. J. Fract. 18 (1982) 237-252.

[9] Tvergaard, V., Needleman, A., Acta Metall. 32 (1984) 157-169.

[10] Peirce, D., Shih, C.F., Needleman, A., Comp. Struct. 18 (1984) 875-878.

[11] Tvergaard, V., J. Mech. Phys. Solids 30 (1982) 399-425. 
[12] Mathur, K.K., Needleman, A., Tvergaard, V., Modelling Simul. Mater. Sci. Eng. 1 (1993) 467484.

[13] Mathur, K.K., Needleman, A., Tvergaard, V., Modelling Simul. Mater. Sci. Eng. 2 (1993) 617635.

[14] Gao, X., Shih, C.F., Tvergaard, V., Needleman, A., J. Mech. Phys. Solids (1996) 44 1255-1282.

[15] Needleman, A., Tvergaard, V., Int. J. Solids Struct. 32 (1995) 2571-2590. 